\title{
Modelo de Canal Relay 3-hop com Realimentação para Sistemas de Altas Taxas
}

\author{
João Luiz Rebelatto e Bartolomeu F. Uchôa-Filho
}

\begin{abstract}
Resumo-O modelo de canal relay introduzido por Meulen, no qual uma fonte e um relay cooperam para enviar a mensagem a um destino, pode gerar um aumento de capacidade quando comparado à transmissão direta em sistemas de comunicação sem fio. Por outro lado, seleção de relay também vem sendo empregada, gerando aumento de capacidade e ganhos de diversidade. Neste trabalho, é proposto um modelo de canal relay 3-hop ao invés do tradicional 2-hop, com seleção dupla de relay, com o intuito de explorar as melhores condições para a transmissão em ambos os links fonte-relay e relay-destino. $O$ esquema proposto considera relays do tipo full duplex e múltiplo acesso por superposição. É mostrado através de simulações computacionais que o esquema 3-hop com dupla seleção proposto pode operar em uma região mais elevada de capacidade que o tradicional modelo 2-hop.
\end{abstract}

Palavras-Chave-Canal relay, capacidade ergódica, comunicação cooperativa, múltiplos usuários.

Abstract-The relay channel model introduced by Meulen, in which a source and relay cooperate to send information to a destination, can provide a capacity increase compared to direct transmission in wireless communication systems. Moreover, relay selection has also been used, generating capacity increase and diversity gains. In this paper a three-hop relay channel model with double relay selection is proposed instead of the tradicional two-hop, in order to exploit the best transmission conditions in both source-relay and relay-destination links. The proposed scheme considers full duplex relaying and superposition multiple access. It is shown through computer simulations that the proposed three-hop scheme with double selection can operate in a higher capacity region than the tradicional two-hop.

Keywords - Relay channel, ergodic capacity, cooperative communication, multiple users.

\section{INTRODUÇÃO}

Os sistemas de comunicação sem fio vêm apresentando um imenso crescimento nos últimos anos. A convergência da tecnologia sem fio com outras tecnologias (como a internet, por exemplo) promete como resultado produtos inovadores e serviços que irão revolucionar vida e trabalho. Associado a isso, a busca por técnicas que aumentem a capacidade dos sistemas de comunicação sem fio vem sendo alvo de uma grande quantidade de pesquisas [1]-[20]. Nesses sistemas sem fio, devido à natureza hostil e aleatória do canal, o emprego de técnicas que melhorem o seu desempenho de erro é de fundamental importância. É também desejável que a taxa de transmissão seja a mais próxima possível da capacidade de canal.

Um modelo de canal introduzido por Van der Meulen [1], denominado canal relay, pode gerar um aumento na capacidade dos sistemas de comunicação. Este canal é composto por

João Luiz Rebelatto e Bartolomeu F. Uchôa-Filho, GPqCom, EEL, Universidade Federal de Santa Catarina, Florianópolis, Brasil, E-mails: \{jlrebelatto,uchoa\}@eel.ufsc.br. Este trabalho foi parcialmente financiado pelo CNPq (142420/2008-5, 303938/2007-2 e 201833/2008-5). três nós: A fonte de informação, o destino e o nó relay, cuja função é cooperar com a transmissão da fonte para o destino, aumentando a sua confiabilidade ou gerando redução na sua potência. Isso se deve ao fato de que geralmente tanto o canal fonte-relay quanto o canal relay-destino apresentam melhores condições (normalmente menores distâncias) para transmissão que o canal fonte-destino. Os relays podem ser utilizados em diversas aplicações, tais como sistemas celulares, redes ad-hoc sem fio e redes de sensores.

Em [2], Cover e El Gamal derivaram expressões exatas para a capacidade do canal relay full duplex sob certas condições e apresentaram limitantes inferiores e superiores para a sua taxa de informação. Outros trabalhos de cunho teórico relacionados a canal relay podem ser destacados [3]-[11]. Estes trabalhos foram desenvolvidos para diferentes cenários: canais Gaussianos [2], [5]-[7], canais de comunicação sem fio ergódicos [4], [7]-[10] e não ergódicos [8], [9], [11], relay do tipo half duplex [8]-[11] e full duplex [2]-[8], e sistemas de comunicação com canal de realimentação [2], [5], [8] e sem canal de realimentação [2]-[4], [9]-[11].

Com o intuito de se aproximar das capacidades apresentadas em [2]-[11], estratégias práticas de codificação e processamento de sinais com relay vêm sendo propostas [12]-[20], inclusive considerando sistemas multiusuário [12]-[15] ou esquemas de seleção (que pressupõe a existência de um canal de realimentação) com uma quantidade maior de relays [18][20], baseados, por exemplo, na aptidão ou não destes para decodificar a mensagem recebida da fonte sem que ocorram erros. Porém, uma característica comum a todos estes trabalhos é o fato de considerarem modelos 2-hop, ou seja, somente um relay é selecionado para auxiliar a transmissão a cada instante de tempo, seguindo o modelo de 3 terminais proposto em [1].

Neste trabalho, partindo do modelo de canal relay 3-hop (com 4 terminais) [21, Cap. 10], ou seja, com a utilização de dois relays capazes de se comunicar entre si, e supondo a existência de um canal de realimentação, é introduzida uma seleção dupla dos dois relays dentre todos os relays disponíveis, de tal forma que sejam extraídas as melhores condições para transmissão tanto no link fonte-relay quanto no link relay-destino. Esta comunicação extra entre os relays caracteriza o modelo 3-hop. Através de simulações computacionais, mostra-se que o modelo proposto, baseado em relays do tipo full duplex [2]-[8] e múltiplo acesso por superposição [22], é capaz de atingir uma região mais elevada de capacidade se comparado com o seu equivalente 2-hop.

O restante do trabalho é organizado como se segue. A Seção II apresenta os principais conceitos do canal relay, assim como os limitantes para sua capacidade, os quais foram expandidos através de conceitos de teoria da informação e obtidos de 
forma gráfica através do método de Monte Carlo [23]. Um modelo de canal relay 3-hop é proposto na Seção III, e são apresentadas suas taxas de informação atingíveis. A Seção IV apresenta resultados de simulações que mostram a eficiência do modelo proposto. Por fim, a Seção V apresenta as conclusões e comentários finais.

\section{Preliminares}

\section{A. Canal relay}

A Figura 1 apresenta o modelo de canal relay definido por Meulen [1].

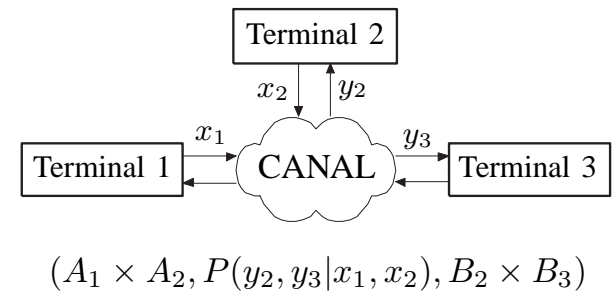

Fig. 1. Modelo de canal relay definido por Meulen [1].

Este modelo é composto por 4 conjuntos, $A_{1}, A_{2}, B_{2}$ e $B_{3}$, e uma quantidade de funções densidade de probabilidade $p\left(\cdot, \cdot \mid x_{1}, x_{2}\right)$ sobre $B_{2} \times B_{3}$, uma para cada $\left(x_{1}, x_{2}\right) \in A_{1} \times$ $A_{2}$. Interpreta-se $x$ como sendo a entrada e $y$ como sendo a saída.

Um exemplo de canal relay full duplex e com múltiplo acesso por superposição está apresentado na Figura 2.

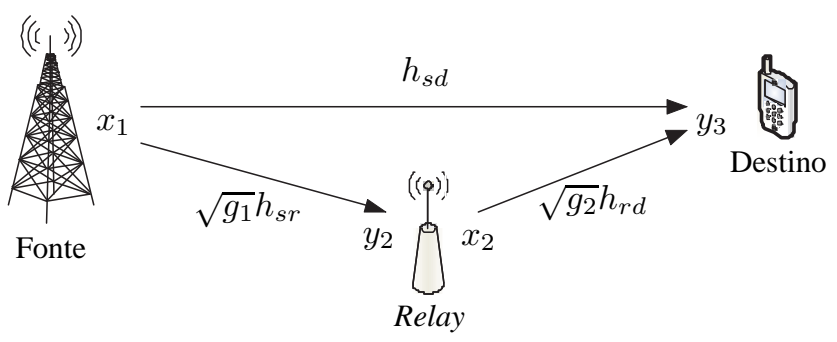

Fig. 2. Exemplo de canal relay full duplex.

Neste modelo, os sinas recebidos no relay e no destino serão, respectivamente:

$$
\begin{aligned}
& y_{2}=\sqrt{g_{1}} \cdot h_{s r} \cdot x_{1}+z_{2} \\
& y_{3}=h_{s d} \cdot x_{1}+\sqrt{g_{2}} \cdot h_{r d} \cdot x_{2}+z_{3}
\end{aligned}
$$

em que $x_{1}$ e $x_{2}$ são os símbolos transmitidos pela fonte e pelo relay, respectivamente. $z_{2}$ e $z_{3}$ representam o ruído Gaussiano, modeladas como variáveis aleatórias complexas i.i.d. com distribuição Gaussiana de média zero e variância $N_{0} / 2$ por dimensão. $g_{1}$ e $g_{2}$ são os ganhos relativos do canal fonte-relay e relay-destino sobre o canal fonte-destino. $h_{s d}$, $h_{s r}, h_{r d}$ são os coeficientes de desvanecimento do canal, modelados como variáveis Gaussianas complexas de média zero e variância unitária. Considera-se que os parâmetros do enlace são conhecidos no destino correspondente, mas não no transmissor.
A cada instante de transmissão, a fonte transmite a sua própria informação. O relay a recebe e, simultaneamente, transmite símbolos baseados na informação recebida da fonte no bloco anterior (depois de decodificá-la e recodificá-la), utilizando a mesma faixa de frequência. $\mathrm{O}$ nó destino recebe uma superposição dos sinais da fonte e do relay, esquema mostrado ser mais eficiente que os ortogonais [22].

$\mathrm{O}$ canal relay combina um canal de radiodifusão (fonte transmitindo para relay e destino) e um canal de múltiplo acesso (fonte e relay transmitindo para o destino). Além do canal relay, pode-se destacar dois métodos de transmissão mais simples: A transmissão direta e a transmissão multihop. $\mathrm{Na}$ transmissão direta, há somente um percurso direto entre a fonte e o destino, isto é, sem nenhuma ajuda do nó relay; na transmissão multihop, há o nó relay, mas a transmissão direta não é considerada. Estas duas maneiras de transmissão mais simples podem servir como referência para avaliar a eficiência do canal relay.

\section{B. Capacidade do canal relay}

Em [2], Cover e El Gamal derivaram limitantes para a capacidade do canal relay full duplex:

$$
\begin{aligned}
& C \leq \max _{p\left(x_{1}, x_{2}\right)} \min \left\{I\left(X_{1}, X_{2} ; Y_{3}\right), I\left(X_{1} ; Y_{3}, Y_{2} \mid X_{2}\right)\right\} \text { (2a) } \\
& C \geq \max _{p\left(x_{1}, x_{2}\right)} \min \left\{I\left(X_{1}, X_{2} ; Y_{3}\right), I\left(X_{1} ; Y_{2} \mid X_{2}\right)\right\}
\end{aligned}
$$

Caso a entrada seja considerada independente e uniformemente distribuída (i.u.d.), a capacidade se reduz à denominada taxa de informação:

$$
\begin{aligned}
& I \leq \min \left\{I\left(X_{1}, X_{2} ; Y_{3}\right), I\left(X_{1} ; Y_{3}, Y_{2} \mid X_{2}\right)\right\} \\
& I \geq \min \left\{I\left(X_{1}, X_{2} ; Y_{3}\right), I\left(X_{1} ; Y_{2} \mid X_{2}\right)\right\}
\end{aligned}
$$

As taxas de informação apresentadas em (3a) e (3b) podem ser claramente visualizadas em dois termos, correspondentes à parte de radiodifusão (BC) e à parte de múltiplo acesso (MAC).

\section{Cálculo da taxa de informação (Monte Carlo)}

Utilizando as propriedades de entropia e informação mútua [24], as taxas de informação apresentadas em (3) podem ser expandidas. Considerando a parte MAC, a expansão da sua taxa de informação resulta em:

$$
\begin{aligned}
I_{M A C} & =I\left(X_{1}, X_{2} ; Y_{3}\right) \\
& =H\left(Y_{3}\right)-H\left(Y_{3} \mid X_{1}, X_{2}\right) \\
& =H\left(Y_{3}\right)-H\left(Z_{3}\right) .
\end{aligned}
$$

Já para a parte BC:

$$
\begin{aligned}
I_{B C}= & I\left(X_{1} ; Y_{3}, Y_{2} \mid X_{2}\right) \\
= & I\left(X_{1} ; Y_{3} \mid X_{2}\right)+I\left(X_{1} ; Y_{2} \mid Y_{3}, X_{2}\right) \\
= & H\left(Y_{3} \mid X_{2}\right)-H\left(Y_{3} \mid X_{1}, X_{2}\right) \\
& \quad+H\left(Y_{2} \mid Y_{3}, X_{2}\right)-H\left(Y_{2} \mid Y_{3}, X_{1}, X_{2}\right) \\
= & H\left(Y_{3} \mid X_{2}\right)-H\left(Z_{3}\right)+H\left(Y_{2}, Y_{3} \mid X_{2}\right) \\
& \quad-H\left(Y_{3} \mid X_{2}\right)-H\left(Z_{2}\right) \\
= & H\left(Y_{2}, Y_{3} \mid X_{2}\right)-H\left(Z_{3}\right)-H\left(Z_{2}\right)
\end{aligned}
$$


Utilizando-se o método de Monte Carlo, de acordo com o apresentado em [23], considerando-se uma grande quantidade de realizações $N$, a entropia de uma variável aleatória $W$ pode ser calculada como sendo:

$$
H(W)=-\frac{1}{N} \sum_{i=1}^{N} \log _{2} p\left(w_{i}\right)
$$

em que $p(w)$ é a distribuição de probabilidade da variável aleatória $W$. A entropia de uma variável Gaussiana com variância $N_{0} / 2$ é conhecida [24] e dada por $H(Z)=\log _{2}\left(\pi e N_{0}\right)$.

A Figura 3 apresenta a taxa de informação (em bits/uso do canal) em função da relação sinal-ruído (em dB) para o sistema com transmissão direta e canal relay, considerando $g_{1}=\infty$ (sem erro de decodificação no relay) e $g_{2}=1$. A potência na transmissão direta foi ajustada de forma que a comparação com o sistema relay pudesse ser feita de maneira justa.

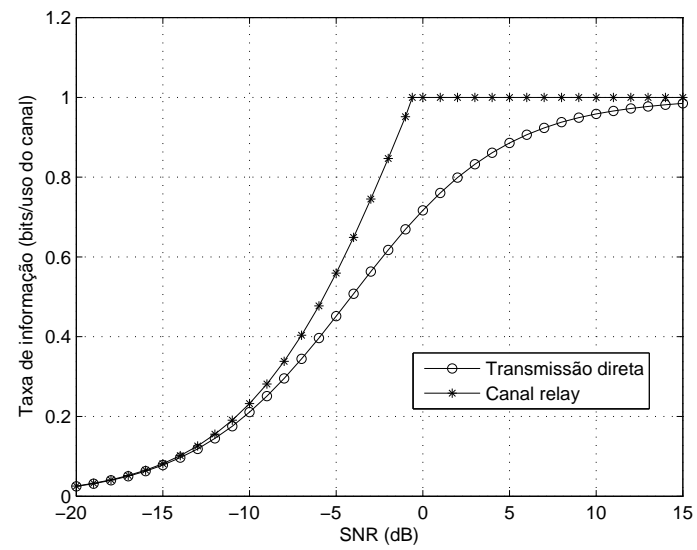

Fig. 3. Taxa de informação (em bits/uso do canal) versus relação sinal-ruído, para transmissão direta e canal relay, considerando $g_{1}=\infty$ e $g_{2}=1$.

A Figura 4 apresenta uma situação mais realista em que $g_{1}=12 \mathrm{~dB}$ e $g_{2}=4 \mathrm{~dB}$. Percebe-se que o canal com relay continua apresentando taxa de informação maior que os outros esquemas.

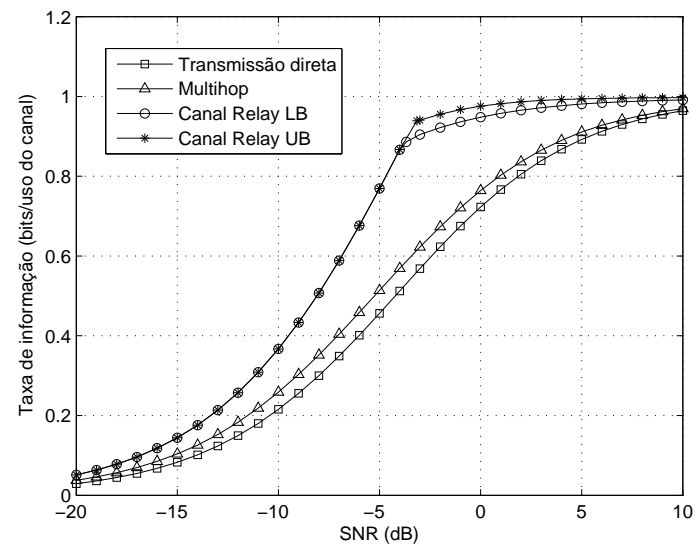

Fig. 4. Taxa de informação (em bits/uso do canal) versus relação sinalruído, para transmissão direta, transmissão multihop e limitantes inferior (LB) e superior (UB) do canal relay, considerando $g_{1}=12 \mathrm{~dB}$ e $g_{2}=4 \mathrm{~dB}$.

\section{Sistema com $n$ relays}

De uma forma mais geral, um sistema pode possuir uma quantidade maior de relays, de acordo com o apresentado na Figura 5. A escolha adequada do(s) relay(s) que será(ão) utilizado(s) na transmissão pode gerar ganhos consideráveis de taxa de transmissão.

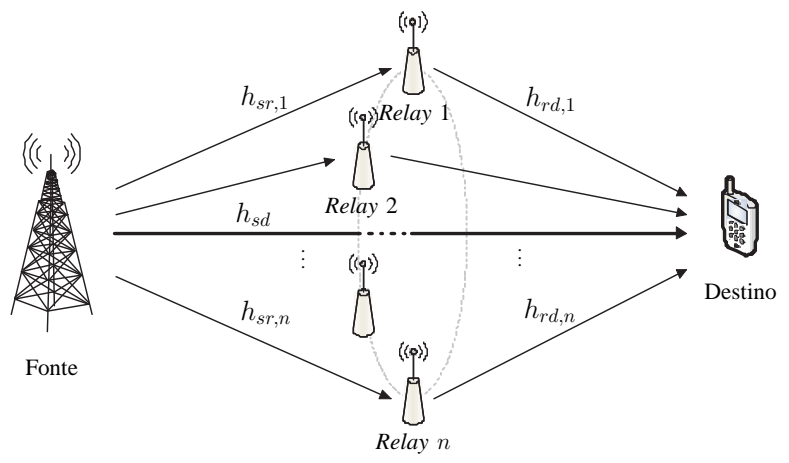

Fig. 5. Modelo de canal com $n$ relays equidistantes da fonte e transmissão direta.

O ganho de taxa de informação gerado pela escolha adequada do relay utilizado na transmissão está ilustrado na Figura 6, para diferentes números de relays $n$. É feita a consideração de que todos os relays são do tipo DAF (decodeand-forward), e o selecionado para a transmissão é o que apresentar melhor canal relay-destino.

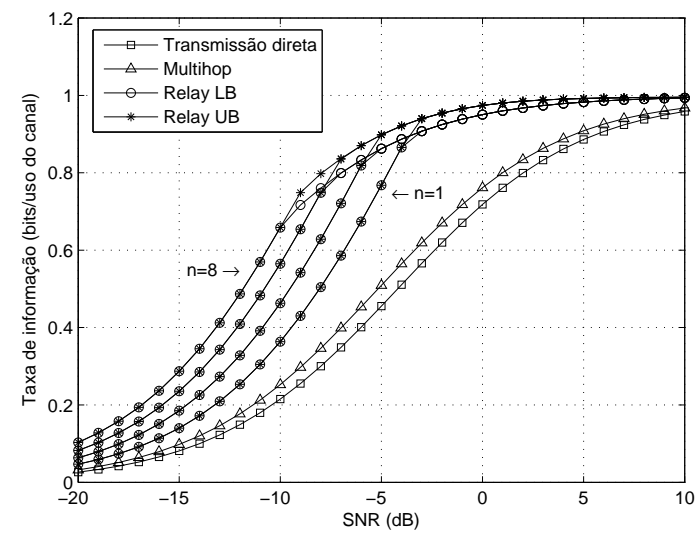

Fig. 6. Taxa de informação (em bits/uso do canal) versus relação sinalruído, para transmissão direta, transmissão multihop e limitantes inferior (LB) e superior (UB) do canal relay, considerando $g_{1}=12 \mathrm{~dB}$ e $g_{2}=4 \mathrm{~dB}$ e número de relays $n=\{1,2,4$ e 8$\}$.

Percebe-se que a taxa de informação cresce com $\log _{2} n$, o que já era esperado devido à analogia deste sistema com sistemas de múltiplas antenas [22].

\section{Sistema Relay 3-hop}

Em um modelo 2-hop, há a seleção de somente um relay para auxiliar na transmissão a cada instante de tempo. Independente do critério que seja utilizado nesta seleção, o relay selecionado muito provavelmente apresentará um dos links (fonte-relay ou relay-destino) em condições que não sejam as melhores possíveis para a transmissão. 
Com o intuito de explorar as melhores condições dos canais fonte-relay e também do canal relay-destino, é proposto o modelo de canal 3-hop com dupla seleção apresentado na Figura 7.

O esquema proposto considera relays do tipo full duplex, os quais, embora ainda possuam restrições de aplicação prática, estão sendo considerados em vários trabalhos [2]-[8], e por apresentar um desempenho (no que diz respeito à capacidade) melhor que os relays do tipo half duplex, devem ser analisados visando aplicações futuras.

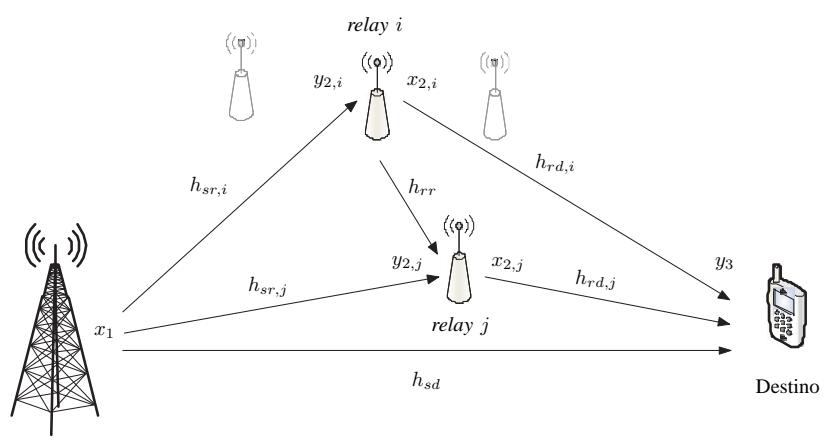

Fonte

Fig. 7. Modelo de canal relay 3-hop proposto.

No esquema proposto, a fonte difunde a informação simultaneamente para o destino e para os relays $r_{i}$ e $r_{j}$, com os melhores canais fonte-relay e relay-destino, respectivamente, ou seja:

$$
\begin{aligned}
r_{i} & =\arg \max _{\eta=1, \ldots, n}\left|h_{s r, \eta}\right|^{2}, \\
r_{j} & =\arg \max _{\eta=1, \ldots, n}\left|h_{r d, \eta}\right|^{2} .
\end{aligned}
$$

em que $n$ é o número de relays e $h_{s r, \eta}$ e $h_{r d, \eta}$ são os coeficientes do canal entre a fonte e o $\eta$-ésimo relay e entre $\eta$-ésimo relay e o destino, respectivamente, ambos com distribuição Rayleigh, compostos por Gaussianas complexas de média zero e variância unitária.

O sinal recebido no relay $r_{i}$ é dado por:

$$
y_{2, i}=\sqrt{g_{1}} \cdot h_{s r, i} \cdot x_{1}+z_{2, i},
$$

em que $g_{1}$ é o ganho relativo do canal fonte-relay sobre o canal fonte-destino, $h_{s r, i}$ é o melhor dentre os $n$ canais fonte-relay, $x_{1}$ é o sinal transmitido pela fonte e $z_{2, i}$ é o ruído Gaussiano no relay $r_{i}$, com média zero e variância $N_{0} / 2$ por dimensão. No instante seguinte, o relay $r_{i}$ transmite o sinal relativo à mensagem recebida no instante anterior simultaneamente para o destino e para o relay $r_{j}$. O sinal recebido no relay $r_{j}$ será a superposição do sinal transmitido pela fonte e pelo relay $r_{i}$ :

$$
y_{2, j}=\sqrt{g_{1}} \cdot h_{s r, j} \cdot x_{1}+\sqrt{g_{r}} \cdot h_{r r} \cdot x_{2, i}+z_{2, j} .
$$

em que $g_{r}$ é o ganho do link $r_{i} \rightarrow r_{j}$ sobre o link de transmissão direta e $h_{r r}$ é o coeficiente do canal entre os relays.

No terceiro instante, $r_{j}$ transmite para o destino o sinal relativo à mensagem recebida de $r_{i}$ no instante anterior (relativo à transmitida pela fonte dois instantes de tempo atrás). A mensagem recebida no destino seria composta pela superposição de três sinais distintos:

$$
y_{3}=h_{s d} \cdot x_{1}+\sqrt{g_{2, i}} \cdot h_{r d, i} \cdot x_{2, i}+\sqrt{g_{2, j}} \cdot h_{r d, j} \cdot x_{2, j}+z_{3},
$$

em que $x_{1}, x_{2, i}$ e $x_{2, j}$ são os símbolos transmitidos pela fonte e pelos relays $r_{i}$ e $r_{j}$, respectivamente. $z_{3}$ representa o ruído Gaussiano no destino, com média zero e variância $N_{0} / 2$ por dimensão. $g_{2, i}$ e $g_{2, j}$ são os ganhos relativos do canal relay $i$-destino e relay $j$-destino sobre o canal fonte-destino. $h_{s d}$, $h_{r d, i}$ e $h_{r d, j}$ são os coeficientes do canal.

Como $x_{2, j}$ é uma função de $x_{2, i}$ no instante anterior, e supondo que o relay $r_{i}$ conhece o esquema de codificação adotado no relay $r_{j}$, a interferência que $x_{2, j}$ causa no relay $r_{i}$ é conhecida e pode, portanto, ser removida. Por essa razão, o canal $r_{j} \rightarrow r_{i}$ é desconsiderado no modelo.

\section{A. Taxa de informação do modelo 3-hop proposto}

De acordo com a teoria de max-flow min-cut [25], para o modelo 3-hop proposto há 4 "cortes", os quais estão apresentados na Figura 8.
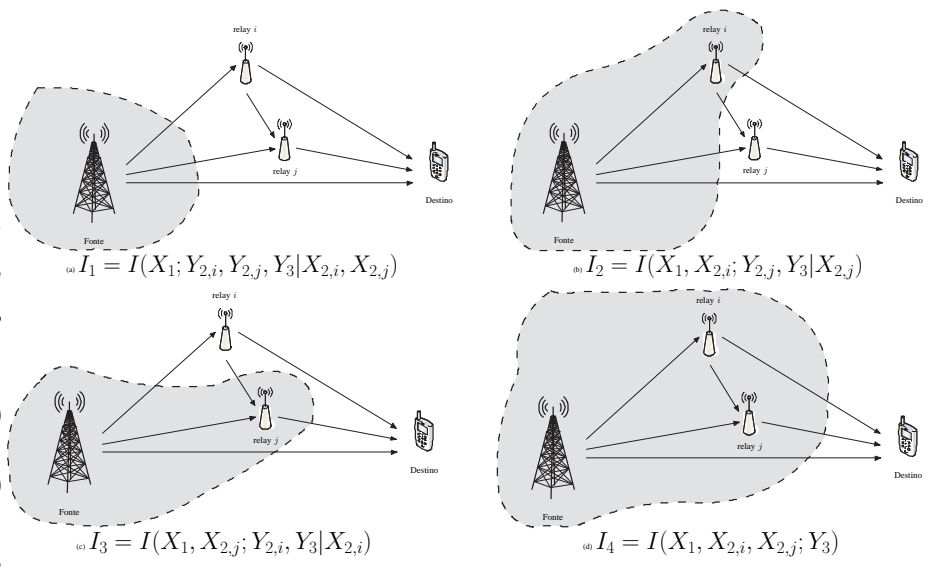

Fig. 8. Max-flow min-cut do modelo 3-hop proposto.

A taxa de informação do sistema será obtida de maneira semelhante à apresentada em [21]:

$$
I=\min \left\{I_{1}, I_{2}, I_{3}, I_{4}\right\} .
$$

Expandindo a taxa de informação do primeiro corte através de conceitos de teoria de informação, obtém-se:

$$
\begin{aligned}
I_{1}= & I\left(X_{1} ; Y_{2, i}, Y_{2, j}, Y_{3} \mid X_{2, i}, X_{2, j}\right) \\
= & H\left(Y_{2, i}, Y_{2, j}, Y_{3} \mid X_{2, i}, X_{2, j}\right) \\
= & \quad-H\left(Y_{2, i}, Y_{2, j}, Y_{3} \mid X_{1}, X_{2, i}, X_{2, j}\right) \\
= & H\left(Y_{2, i}, Y_{2, j}, Y_{3} \mid X_{2, i}, X_{2, j}\right) \\
& \quad-H\left(Z_{2, i}\right)-H\left(Z_{2, j}\right)-H\left(Z_{3}\right) .
\end{aligned}
$$

De maneira semelhante, pode-se obter a taxa de informação dos outros cortes, que são dadas por:

$$
\begin{aligned}
I_{2} & =I\left(X_{1}, X_{2, i} ; Y_{2, j}, Y_{3} \mid X_{2, j}\right) \\
& =H\left(Y_{2, j}, Y_{3} \mid X_{2, j}\right)-H\left(Z_{3}\right)-H\left(Z_{2, j}\right) . \\
I_{3} & =I\left(X_{1}, X_{2, j} ; Y_{2, i}, Y_{3} \mid X_{2, i}\right) \\
& =H\left(Y_{2, i}, Y_{3} \mid X_{2, i}\right)-H\left(Z_{3}\right)-H\left(Z_{2, i}\right) .
\end{aligned}
$$




$$
\begin{aligned}
I_{4} & =I\left(X_{1}, X_{2, i}, X_{2, j} ; Y_{3}\right) \\
& =H\left(Y_{3}\right)-H\left(Y_{3} \mid X_{1}, X_{2, i}, X_{2, j}\right) \\
& =H\left(Y_{3}\right)-H\left(Z_{3}\right) .
\end{aligned}
$$

Ressalta-se que diferentemente do esquema com 2 relays proposto em [21], o esquema proposto neste trabalho considera os canais fonte- $r_{i}$ e $r_{j}$-destino como sendo order statistics, e que para este tipo de canal, não foi encontrado na literatura trabalhos que utilizem 2 ou mais relays.

\section{Resultados das Simulações}

Na Figura 9 está apresentado o limitante superior para a taxa de informação atingível com o modelo 3-hop proposto, aplicando-se o método de Monte Carlo às equações (12)-(16) e considerando uma quantidade de $n=4$ relays, $g_{1}=12 \mathrm{~dB}$ e $g_{2, i}=g_{2, j}=4 \mathrm{~dB}$. Além disso, a Figura 9 também apresenta o limitante superior para a taxa de informação do modelo de canal 2-hop com $n=4$ relays (apresentada inicialmente na Figura 6). Para que fosse realizada uma comparação justa, a potência de transmissão da fonte e dos relays do modelo 3-hop proposto foram reduzidas por um fator $2 / 3$, de tal forma que a potência total de transmissão de ambos os esquemas, assim como da transmissão direta e multihop, fosse a mesma.

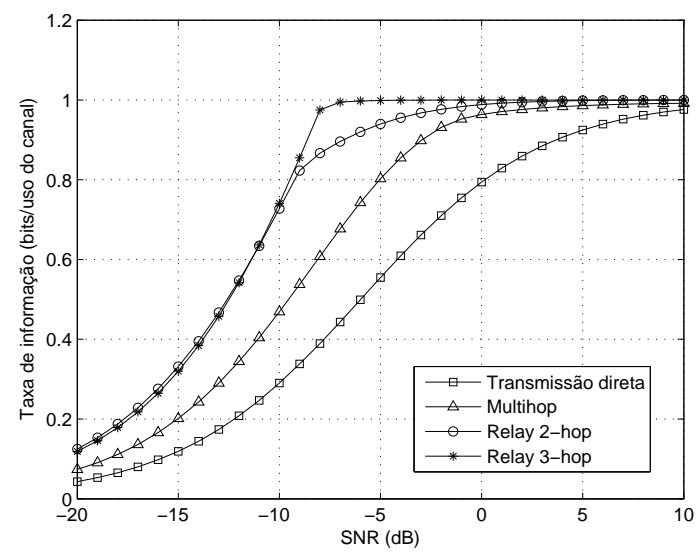

Fig. 9. Taxa de informação versus SNR, para $g_{1}=12 \mathrm{~dB}$ e $g_{2, i}=g_{2, j}=4$ $\mathrm{dB}$, considerando a transmissão direta, transmissão multihop, canal relay 2hop e o modelo 3-hop proposto, ambos para $n=4$ relays.

Deve-se notar que, para altas taxas $(>0,85)$, o ganho de SNR é significativo. Por exemplo, para uma taxa de 0,9 bits/uso do canal, o ganho é de aproximadamente $2,5 \mathrm{~dB}$ em relação ao esquema relay 2-hop.

Quando o número de relays $n$ torna-se maior, maior também é o ganho obtido em altas taxas pelo sistema 3-hop em relação ao 2-hop, de acordo com o apresentado nas Figuras 10 e 11, considerando $n=32$ e $n=512$, respectivamente.

Para $n=32$ relays, o ganho obtido com o sistema 3hop, considerando uma taxa de 0,9 bits/uso do canal é de aproximadamente $4 \mathrm{~dB}$. Se considerarmos $n=512$, esse ganho sobe para mais de $5 \mathrm{~dB}$.

Deve-se levar em consideração que o sistema 3-hop apresenta uma latência (atraso) maior que o 2-hop, uma vez que a mensagem transmitida pela fonte em dado instante demorará

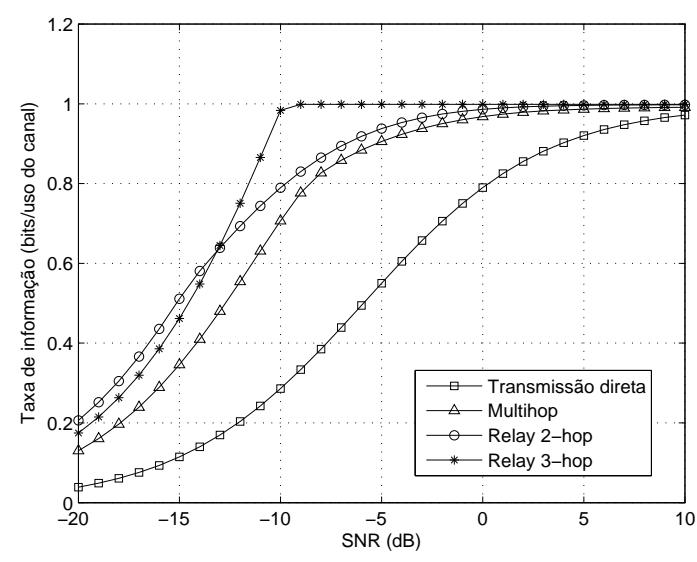

Fig. 10. Taxa de informação versus SNR, para $g_{1}=12 \mathrm{~dB}$ e $g_{2, i}=$ $g_{2, j}=4 \mathrm{~dB}$, considerando a transmissão direta, transmissão multihop, canal relay 2-hop e o modelo 3-hop proposto, ambos para $n=32$ relays.

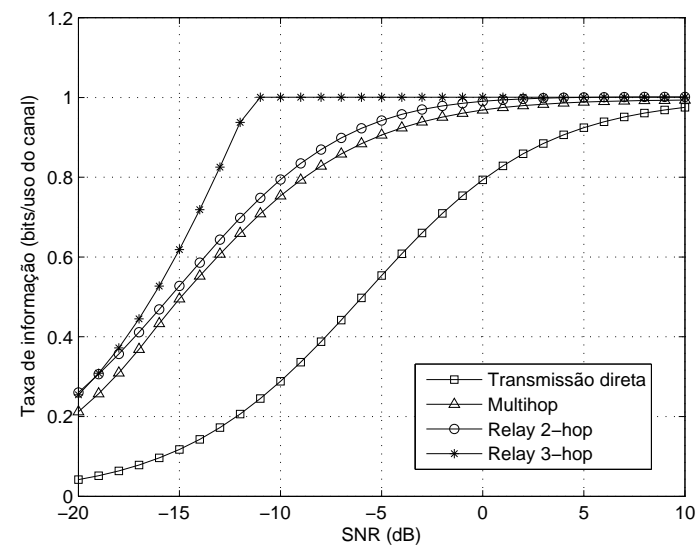

Fig. 11. Taxa de informação versus SNR, para $g_{1}=12 \mathrm{~dB}$ e $g_{2, i}=$ $g_{2, j}=4 \mathrm{~dB}$, considerando a transmissão direta, transmissão multihop, canal relay 2-hop e o modelo 3-hop proposto, ambos para $n=512$ relays.

um instante de tempo (aqui tratado como o tempo necessário para a transmissão de um bloco de símbolos, ou frame) a mais para estar completamente disponível no destino.

\section{CONCLUSÕES}

Um esquema de canal relay com realimentação capaz de atingir uma taxa de informação mais elevada foi apresentado. $\mathrm{O}$ esquema proposto é baseado em um modelo 3-hop ao invés do tradicional 2-hop. O modelo se aplica à situação prática em que um nó fonte transmite informação a um nó destino distante e, em uma região geográfica intermediária, $n$ nós relays encontram-se próximos entre si e disponíveis para cooperar. Mostrou-se através de simulações que ganhos maiores que $5 \mathrm{~dB}$ podem ser obtidos com o esquema proposto. Com o intuito de aproximar a taxa de informação obtida na prática dessas taxas de informação teóricas, estratégias de codificação de canal baseadas em códigos turbo e LDPC, na linha de [26]-[28], serão elaboradas na seqüência do trabalho e associadas ao modelo 3-hop proposto. 


\section{REFERÊNCIAS}

[1] E. C. van der Meulen, "Three-terminal communication channels," $A d$ vanced Applied Probability, vol. 3, pp. 120-154, 1971.

[2] T. M. Cover and A. E. Gamal, "Capacity theorems for the relay channel," IEEE Trans. Inf. Theory, vol. 25, no. 9, pp. 572-584, Setembro 1979.

[3] P. Vanroose and E. van der Meulen, "Uniquely decodable codes for deterministic relay channels," IEEE Trans. Inf. Theory, vol. IT-38, no. 4, pp. 1203-1212, Julho 1992.

[4] G. Kramer, M. Gastpar, and P. Gupta, "Cooperative strategies and capacity theorems for relay networks," IEEE Trans. Inf. Theory, vol. 51, no. 9, pp. 3037-3063, Setembro 2005.

[5] L. L. Xie and P. R. Kumar, "An achievable rate for the multiple-level relay channel," IEEE Trans. Inf. Theory, vol. 51, no. 4, pp. 1348-1358, Abril 2005.

[6] A. Reznik, S. R. Kulkarni, and S. Verdu, "Degraded gaussian multirelay channel: Capacity and optimal power allocation," IEEE Trans. Inf. Theory, vol. 50, no. 10, pp. 3037-3046, Dezembro 2004.

[7] B. Wang, J. Zhang, and A. Host-Madsen, "On the capacity of MIMO relay channels," IEEE Trans. Inf. Theory, vol. 51, no. 1, pp. 29-43, Janeiro 2005

[8] A. Host-Madsen and J. Zhang, "Capacity bounds and power allocation for wireless relay channels," IEEE Trans. Inf. Theory, vol. 51, no. 6, pp. 2020-2040, Junho 2005.

[9] R. U. Nabar, H. Bölcskei, and F. W. Kneubühler, "Fading relay channels: Performance limits and space-time signal design," IEEE J. Sel. Areas Commun., vol. 22, no. 6, pp. 1099-1109, Agosto 2004.

[10] G. Farhadi and N. C. Bealieu, "On the capacity of wireless relaying systems over rayleigh fading channels," IEEE Trans. Wireless Commun., vol. 7, no. 11, pp. 4462-4467, Novembro 2008.

[11] H. Bolcskei, R. U. Nabar, O. Oyman, and A. J. Paulraj, "Capacity scaling laws in MIMO relay networks," IEEE Trans. Wireless Commun., vol. 5, no. 6, pp. 1433-1444, Junho 2006.

[12] A. Sendonaris, E. Erkip, and B. Aazhang, "User cooperation diversity: Part I and Part II," IEEE Trans. Commun., vol. 51, no. 11, pp. 19271948, Novembro 2003.

[13] J. N. Laneman, D. N. C. Tse, and G. W. Wornell, "Cooperative diversity in wireless networks: Efficient protocols and outage bahavior," IEEE Trans. Inf. Theory, vol. 50, no. 12, pp. 3062-3080, Dezembro 2004.
[14] T. E. Hunter and A. Nosratinia, "Cooperative diversity through coding," in Proc. IEEE International Symposium on Information Theory, ISIT'02, Lausanne, Switzerland, Julho 2002, p. 220.

[15] A. Stefanov and E. Erkip, "Cooperative coding for wireless networks," IEEE Trans. Commun., vol. 52, no. 9, pp. 1470-1476, Setembro 2004.

[16] — "Cooperative space-time coding for wireless networks," IEEE Trans. Commun., vol. 53, no. 11, pp. 1804-1809, Novembro 2005.

[17] Z. Liu, V. Stankovic, and Z. Xiong, "Wyner-ziv coding for the halfduplex relay channel," in Proc. IEEE International Conference on ASSP, Philadelphia, PA, Março 2005, pp. 1113-1116.

[18] Y. Zhao, R. Adve, and T. J. Lim, "Improving amplify-and-forward relay networks - optimal power allocation versus selection," IEEE Trans. Wireless Commun., vol. 6, no. 8, pp. 3114 - 3123, Agosto 2007.

[19] E. Beres and R. Adve, "On selection cooperation in distributed networks," in Proc. IEEE Conference on Information Sciences and Systems. CISS'06, Março 2006, pp. 1056-1061.

[20] Y. Li, B. Vucetic, Z. Chen, and J. Yuan, "An improved relay selection scheme with hybrid relaying protocols," in Proc. IEEE Global Telecommunications Conference. GLOBECOM '07, Novembro 2007, pp. 3704 $-3708$.

[21] G. Kramer, Topics in Multi-User Information Theory. Foundations and

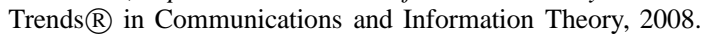

[22] D. Tse and P. Viswanath, Fundamentals of Wireless Communications. Cambridge: Cambridge University Press, 2005.

[23] D. Arnold and H.-A. Loeliger, "On the information rate of binary-input channels with memory," in Proc. IEEE International Conference on Communications. ICC'01, vol. 9, Junho 2001, pp. 2692-2695.

[24] T. M. Cover and J. A. Tomas, Elements of Information Theory. Nova Iorque: John Wiley \& Sons, 1991.

[25] L. R. Ford and D. R. Fulkerson, "Maximal flow through a network," Canadian Journal of Mathematics, vol. 8, pp. 399-404, 1956.

[26] Z. Zhang and T. M. Duman, "Capacity approaching turbo coding and iterative decoding for relay channels," IEEE Trans. Commun., vol. 53, no. 11, pp. 1895-1905, Novembro 2005.

[27] — "Capacity approaching turbo coding for half duplex relaying," in Proc. IEEE International Symposium on Information Theory. ISIT'05, Adelaide, Australia, Setembro 2005, pp. 1888-1892.

[28] J. Hu and T. M. Duman, "Low Density Parity Check codes over wireless relay channels," IEEE Trans. Wireless Commun., vol. 6, no. 9, pp. 3384 3394, Setembro 2007. 